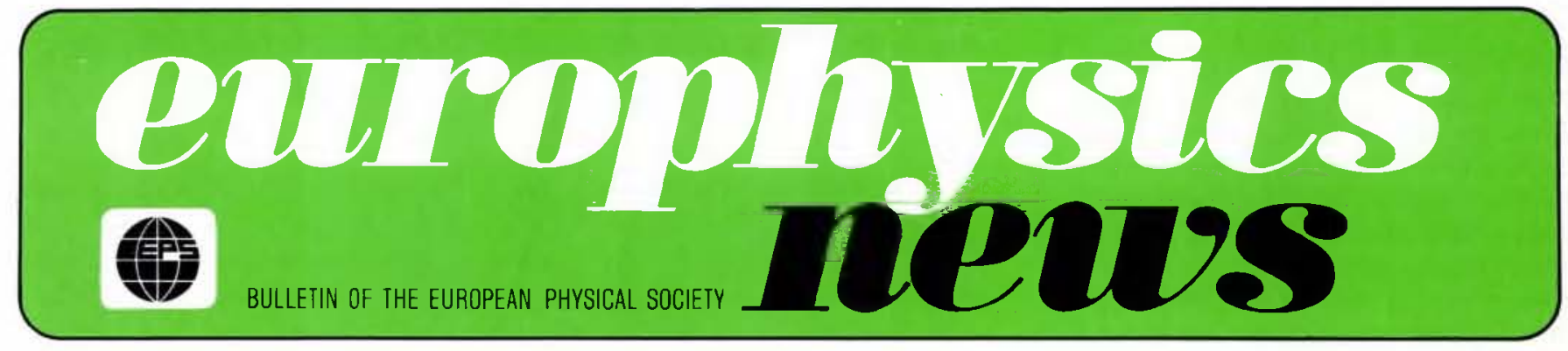

\title{
Generation and Applications of Femtosecond Laser Pulses
}

\author{
S. De Silvestri, P. Laporta and V. Magni, Milan
}

(Centro di Elettronica Quantistica e Strumentazione Elettronica del CNR)

Since the invention of the laser, a rapid and continuous advance has been taking place in the generation of short light pulses running closely parallel to the development of new laser sources and the introduction of innovative concepts and techniques. The first significant step came in 1966 with the generation of pulses shorter than $10 \mathrm{ps}\left(10^{-11} \mathrm{~s}\right)$ followed in 1968 by both a decrease in pulse width (a few picoseconds) and a wide extension of the wavelength range. In 1972 , the width was reduced to $1.5 \mathrm{ps}$ and then in 1981 to less than $100 \mathrm{fs}$ $\left(10^{-13} \mathrm{~s}\right)$. Since then, pulses as short as 8 fs have been produced.

With the availability of shorter light pulses and new measuring techniques, most of which rely on non-linear optical interactions, a large variety of physical phenomena can now be investigated, and new research areas have been opened up. The study of previously unresolved, fast processes in physics, chemistry and biology has led to the discovery of interesting phenomena, which shed new light on the dynamic properties of matter.

\section{Femtosecond Pulse Generation}

Generating ultrashort light pulses requires a laser to operate in a particular regime, called mode-locking which may be illustrated either in the frequency or the time domain. Depending on the particular case, one description is much more intuitive than the other and we have chosen to present the simpler approach.

A typical laser consists of an optical resonator, made up of either plane or curved mirrors, enclosing the laser gain medium. The electromagnetic field inside can be described in terms of cavity modes, characterized by particular field distributions and regularly spaced resonance frequencies. Oscillation of the modes is sustained within the laser cavi-

Fig. 1 - Schematic of pulse-shortening mechanism in passively mode-locked dve lasers. The dashed pulse is a replica of the pulse entering the saturable absorber. The pulse shortening per round trip is given by $\delta \tau=\tau-\tau^{\prime}$.

\section{SATURABLE ABSORBER}

\section{SATURABLE GAIN}

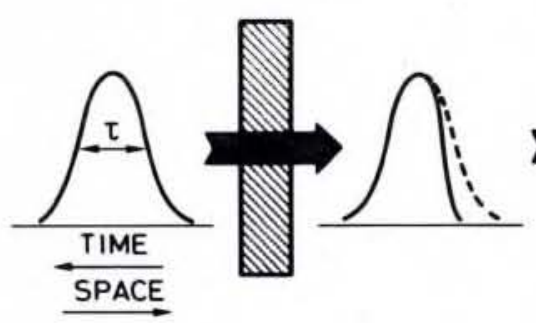

LINEAR

LOSSE S

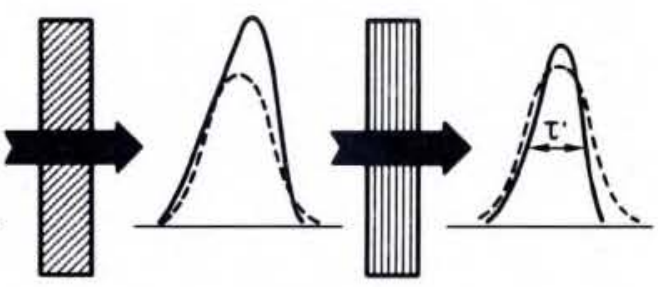

ty when the gain exceeds the total losses. Consequently the number of modes depends upon the spectral width of the gain medium. In general, there is no well-defined phase and amplitude relationship between different modes and only when they are forced to main tain one will the laser output vary in a well-defined and predictable manner. Such laser operation is called modelocking. It produces a train of short light: pulses whose duration is inversely proportional to the total oscillating bandwidth. The time between two successive pulses is equal to the round-trip time of the resonator.

Locking of the modes may be achieved through active or passive modula-

\section{Contents}

Generation and Applications of Femtosecond Laser Pulses

Non-classical Light

Frustrated Liquids:

Liquid Crystal Blue Phases

Laser-Generated Intense

Planck Radiation

EPS Publications

EPS Staff 
tion of cavity losses and/or gain. In active mode-locking the modulation is introduced by an external energy source, whereas in passive mode-locking the system provides its own modulation. Mode-locking has been applied to solidstate lasers, gas lasers, dye lasers, semiconductor lasers and colour centre lasers. However, only laser media with broad energy levels, such as dye molecules, semiconductors and colour centres, offer the possibility of obtaining ultrashort pulses and since the shortest pulses have to date been generated only in $\mathrm{CW}$ dye lasers using passive modelocking, we confine ourselves to illustrating in detail this particular technique.

Passive mode-locking involves the use of an intra-cavity saturable absorber, a material whose transmission increases with the energy absorbed. The dynamics of pulse shortening can be easily explained by time domain analysis, as shown in Fig. 1. For the sake of simplicity, we assume a pulse already built up inside the laser cavity. As the pulse enters the absorber, its leading edge is strongly absorbed, while the peak and the trailing edge pass almost unchanged, owing to absorption saturation. When the pulse passes through the gain medium, the gain saturates, and the gain experienced by the trailing edge is reduced. Losses reduce the overall pulse energy to its former value. In short, pulse reshaping consists in cutting the tails and enhancing the peak amplitude. Within certain limits a constant energy pulse can be progressively shortened with each round trip. A pulse-shortening velocity per round trip can be defined ${ }^{1}$ ) as: $S=$ $\delta \tau / \tau$, where $\delta \tau$ is the pulse-shortening per pass and $\tau$ is the pulse duration.

The mechanism described above would generate progressively shorter pulses were it not for mechanisms responsible for broadening. These are mainly represented by two linear effects $\left.{ }^{2}\right)$ : the filtering action of bandwidthlimiting elements (such as gain bandwith, mirrors, tuning elements) and group-velocity dispersion (such as material dispersion and reflection at dielectric mirrors). Finally, a complicated nonlinear phenomenon called self-phase modulation, may also affect the pulse and may even be exploited for pulse shortening. The filtering action narrows the pulse bandwidth and therefore increases the pulse width, as the two are inversely proportional while group-velocity dispersion does not affect the spectral bandwidth, but modifies the relative phases of the harmonic waves forming the pulses. This process broadens the pulse envelope and generates a carrier
Fig. 2 - Steady-state pulse duration of a mode-locked laser cavity as a function of the bandwidth. The dashed curve represents the dispersionless case and the solid curves show the increase in pulse duration for different values of the dispersion parameter lexpressed in units of $\left.\mathrm{fs} / \mathrm{cm}^{-1}\right)$.

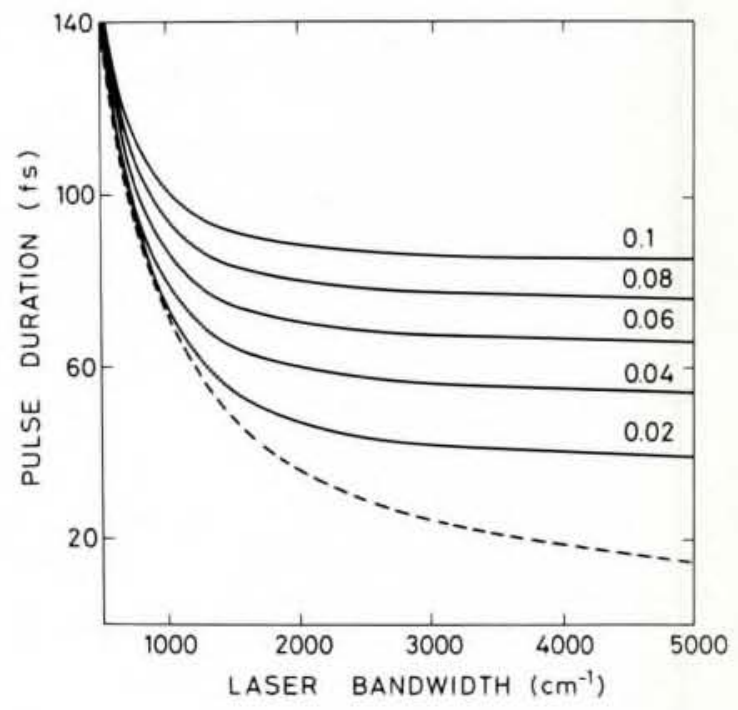

frequency sweep. The effects of filtering and dispersion can be simply combined into a pulse-broadening velocity which at minimum pulse duration balances the pulse-shortening velocity. Assuming for $S$ a typical value of $1 \%$, Fig. 2 shows pulse duration as a function of laser bandwidth for different amounts of dispersion introduced by reflection upon the dielectric mirrors of the cavity ${ }^{3,4}$ ). It is apparent that the effect of dispersion is to limit the minimum pulse duration attainable, irrespective of the laser bandwidth.

Progress in generating ultrashort pulses came through

(i) an increase in pulse-shortening velocity;

(ii) expansion of filter bandwidth;

(iii) compensation for dispersion; and (iv) taking advantage of the self-phase modulation effect in the cavity media.

High pulse-shortening velocity was achieved by the introduction of the CPM ring-dye laser ${ }^{5}$ ) wherein two counterpropagating pulses collide in the saturable absorber (Fig. 3), which thus becomes more effective in pulse shortening. Expansion of the spectral bandwidth can be simply achieved by eliminating all bandwidth-limiting elements inside the

cavity, leaving the mirrors as the ultimate limiters. This implies that, at present, ultrashort light pulses are virtually untunable. Dispersion caused by reflection from dielectric mirrors, as well as material dispersion (dye solvent, quartz, etc.) can be offset by using pairs of prisms inside the cavity, which introduce dispersion of the opposite sign.

In both the saturable absorber and the gain medium, the high intensity of the light pulse may change the material refractive index, which, in turn, modifies the phase of the optical carrier, giving rise to a frequency sweep (self-phase modulation, SPM). Since SPM is a nonlinear phenomenon, new frequencies are added to the pulse spectrum. Combination of SPM and dispersion can be exploited to broaden the pulse band width and thus compress pulse duration. Pulses as short as $27 \mathrm{fs}(100 \mathrm{MHz}$ repetition rate at $620 \mathrm{~nm}$ wavelength) have been generated in a CPM laser with two pairs of prisms inside the cavity that exactly compensate for SPM and dispersion ${ }^{6}$ ).

Although the high repetition rate makes it possible to perform a variety of experiments, the investigation of nonlinear optical effects often requires high

Fig. 3 - Schematic of a CPM ring dye laser.

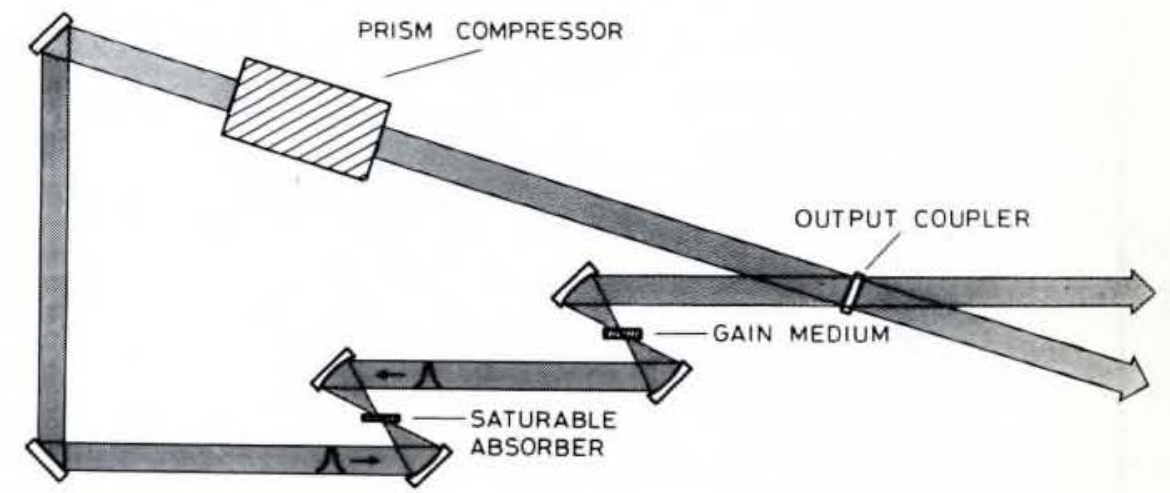


peak power. Multi-stage dye amplifiers have been built to raise the pulse peak power from about one kilowatt (as obtained with CPM femtosecond lasers) to the gigawatt level. The design of these systems must be quite accurate, in order to avoid or compensate for any pulsebroadening effects. By focussing amplified femtosecond pulses into non-linear optical materials, it is possible to generate, mainly by SPM, white-light continuum pulses with a wavelength content ranging from the near ultraviolet to the near infrared ${ }^{7}$ ). This large spectral broadening makes for more effective pulse compression outside the laser cavity where the shortest light pulses so far obtained, 8 fs (only four optical cycles!) have been generated ${ }^{8}$ ).

\section{Measurement Techniques}

Conventional light detector and electronics have a time resolution that is limited to a few picoseconds. For measuring femtosecond pulses, or for studying the dynamics of their interactions with matter, the only techniques available rely on non-linear optical interactions ${ }^{9}$ ). Autocorrelation measurements by second harmonic generation are so far the commonest means of evaluating duration. The light pulse is split and one pulse is used to "sample" the other with a variable delay. This is achieved by crossing the two pulses in a non-linear crystal, which generates a second harmonic signal proportional to the product of the two pulse intensities. The delay between the two pulses is varied by changing the optical path of one. By measuring the second harmonic intensity as a function of the delay, the autocorrelation function of the pulse temporal profile is obtained, and this enables pulse duration to be determined. Such measurements require an extremely accurate variable delay (path length variations of the order of $0.1 \mu \mathrm{m})$ and the use of non-dispersive optics, with for example metal instead of dielectric mirrors.

The same experimental scheme is also used for studying ultrashort processes, the non-linear crystal being replaced by the sample under study. One of the two pulses, called the pump pulse, is used to excite the sample, the second, called the probe, is detected by a slowresponse detector. The recovery of the sample transmission is thus monitored as a function of the delay from the pump. The pump-probe method has been refined and extended, and remains the most versatile and most widely used technique for high temporal resolution measurements. An extension of the technique is three-pulse scattering in which transient absorption or a phase-grating is established by interference of two time-coincident pulses crossed inside the sample. A third pulse with a variable time delay scatters off the grating, and the scattered energy, measured as a function of delay, provides information on the grating dynamics, and hence on the processes occurring inside the material.

\section{Applications}

Femtosecond laser pulses are finding wide areas of application in physics, chemistry, and biology. Three properties make them especially useful: short duration, large bandwidth, and short spatial length. High temporal resolution is particularly important for measuring relaxation processes in molecules, as well as transport and relaxation phenomena in solid-state materials. The large bandwidth of femtosecond pulses and the white light continuum may also give birth to a new spectroscopy. The short spatial length may lead to high-resolution optical ranging as a non-destructive technique for diagnostic analysis of solid-state and biological materials.
The range of femtosecond laser applications is too wide to attempt a comprehensive review. We therefore limit ourselves to a few specific examples, which indicate how the field is spreading into many different research areas.

Non-radiative relaxation processes are extremely fast in organic molecules and in biological material. Pump-probe techniques have been applied to show their sub-picosecond nature in a number of different samples. The ultrafast orientational momentum and energy relaxation of highly-excited electrons in the conduction band of semiconductors has also been studied ${ }^{10}$ ). Experiments performed in GaAs and AIGaAs reveal that photo-excited electrons decay in less than $100 \mathrm{fs}$, owing to electron-electron and optical phonon scattering. A knowledge of these processes is of primary importance in understanding the dynamics of short-pulse formation in semiconductor lasers, and the time response of optical non-linearities in semiconductors.

A new technique has been applied to filming the progress of an ultrafast melt and material ejection from a semicon-

\section{Institut de Physique Nucléaire
Louvain-la-Neuve Belgium
Staff position in}

The Institut de Physique Nucléaire (IPN) wishes to appoint a young experimental nuclear and/or particle physicist to a tenured staff position. The appointment will be with the Catholic University of Louvain, in the Permanent Scientific Personnel. It is anticipated to be open by September 1987.

The IPN is a research institute in experimental nuclear and particle physics, jointly sponsored by the Catholic University of Louvain (UCL) and the Interuniversity Institute for Nuclear Sciences (IISN) which is Belgium's funding agency for fundamental nuclear sciences in universities.

The main facility of the IPN is a $\mathrm{K}=120 \mathrm{MeV}$ isochronous cyclotron for the acceleration of light and heavy ions, equipped with a high-performance Electron Cyclotron Resonance (ECR) heavy-ion source, and producing a wide variety of high-intensity beams, presently up to $X e$. Equipment for research around the cyclotron includes various systems for the detection and spectroscopy of neutrons, light and heavy ions, electrons (with positron polarimetry) and $\gamma$-rays (with Compton suppression), an online isotope separator (LISOL) with a low-temperature nuclear orientation device (KOOL). There is also a $4 \mathrm{MV}$-van de Graaff mainly used for implantation studies with polarized nuclei. The Institute is involved in several experiments at large facilities outside Belgium, in heavy ion physics (at GANIL, Caen, and SARA, Grenoble) and in elementary particle physics (at CERN, Geneva, and SIN, Villigen).

The candidates must be interested in the research performed by the Institute, more specifically in heavy ion physics and/or elementary particle physics; several years of post-doctoral experience are required. An elementary knowledge of French is welcome; the candidates will have to learn it up to the level needed for teaching duties. Applicants for the above-mentioned position are requested to submit a "curriculum vitae", list of publications, indications of their teaching experience, and the names and addresses of three references, as early as possible but not later than 15 December 1986, to:

Professor J. Vervier, Institut de Physique Nucléaire, Université Catholique de Louvain, Chemin du Cyclotron, 2, B - 1348 Louvain-la-Neuve, Belgium. 


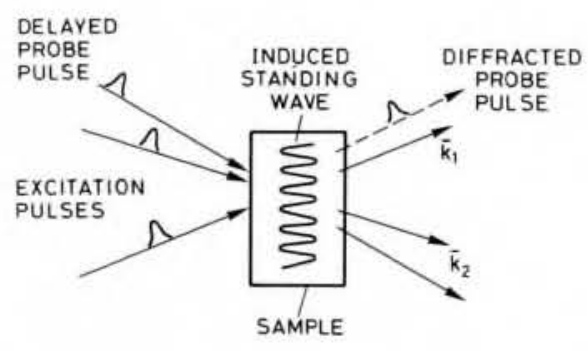

Fig. 4 - Interaction scheme for optical phonon "impulsive" excitation and detection.

ductor surface, ${ }^{11}$ ). Using amplified femtosecond pulses, a pump beam was focussed onto a silicon surface and a white light continuum pulse used to probe the area excited by the pump beam. $A$ magnified image of the excited region was obtained at a given pump-probe delay by collecting the specularly reflected continuum light. The series of images obtained provide a detailed picture of the physics of a highly excited semiconductor surface as a function of time.

The coherence of femtosecond pulses makes it possible to investigate transient coherent phenomena, and to separate dephasing from energy relaxation $\left.{ }^{12}\right)$. The method relies on a modified version of the three-pulse scattering technique, based on an optically-induced grating, formed by the interference of two pulses in the sample. When the two pulses are separated temporally, a grating can still be formed, provided that the sample's memory (i.e. the dephasing time) of the arrival of the first pulse is sufficiently long. By measuring the grating amplitude as a function of the delay of the two pulses, one can measure the dephasing time. This is accomplished by using a third pulse as a delayed probe. The technique has been applied to the dephasing of dye molecules in a polymer host at low temperature.

The study of optical phonon interactions in crystals is of considerable importance because of the central role they play in redistributing lattice energy. Excitation and detection of low-frequency coherent optic phonons in solids can be achieved in a very original and elegant fashion by means of femtosecond optical pulses ${ }^{13}$ ). Two pulses (see Fig. 4), with the same frequency and wavevectors $\mathbf{k}_{1}$ and $\mathbf{k}_{2}$ are crossed in a sample to excite coherent optic phonons with the difference wave-vectors $\pm\left(\mathbf{k}_{1}\right.$ $\mathbf{k}_{2}$ ). The counter-propagating phonons form a vibrational standing wave whose frequency is determined by the phonon frequency. Successful use of this technique requires the pulse duration to be shorter than the time of a single vibrational period so that the vibrational mode is driven "impulsively". With pulses of less than $100 \mathrm{fs}$, optic phonons of wave number up to $200 \mathrm{~cm}^{-1}$ can be investigated. The standing wave character of the material excitation permits measurement of the phonon frequency and damping through direct observation using a variably delayed probe pulse which coherently scatters off the grating. Fig. 5 shows the decay curves of such optical phonons in a molecular crystal at low temperature as a function of the probe delay. The simultaneous excitation of two optic phonons accounts for the beating observed in the decay. This is the first observation of optic phonon oscillations directly in the time domain.

For a pulse lasting $100 \mathrm{fs}$, the spatial length of the photon packet is about 30 $\mu \mathrm{m}$ and this can be used effectively for gated picture ranging ${ }^{14} \mathrm{H}$. The basic scheme consists of sending a probe pulse toward the target of interest and recording the back-scattered pulses by means of non-linear mixing with a reference pulse. The experimental set-up is similar to that used for second harmonic autocorrelation measurements. The desired echo pulses can be resolved by judicious gating with the reference pulse. The spatial resolution in only limited by the pulse duration. Femtosecond optical ranging has been demonstrated in biological systems by investigating the cornea of rabbits eyes in vivo and the structure of human skin.

Femtosecond pulses have also been used to sample electrical signals generated by high-speed, solid-state electronic devices ${ }^{15}$ ). The sampling technique is based on the electro-optic effect, i.e. the ability of some crystals to change their birefringence, and hence the polarization of the transmitted light, as the applied electric field is varied. Schematically the electro-optic sampling system is formed by placing a crystal between two crossed polarizers. The transmission changes of a variably-delayed optical pulse train, brought about by fieldinduced birefringence, provide a "gated" measurement of the electric signal applied. Electro-optic sampling with less than $100 \mathrm{fs}$ pulses has been employed to measure electrical transients with 300 fs temporal resolution.

\section{Conclusions}

Rapid progress is being made in the field of femtosecond pulse generation. The fundamental physical limit for minimum pulse duration in the visible spectral range is fast approaching, and pulses of the order of the period of an optical cycle are not far off. At the same time, research on conventional and new laser sources is becoming very promising for generating femtosecond optical pulses over a wider spectral range. The application of ultrashort light-pulses in an increasing variety of research areas is making it possible to develop new experimental tools and concepts that will certainly contribute to further advances in science and technology.

\section{REFERENCES}

1. Stix M. and Ippen E.P., IEEE J. Quantum Electron. QE-19 (1983) 520.

2. Silvestri S. De, Laporta P. and Svelto $O$., IEEE J. Quantum Electron. QE-20 (1984) 533.

3. Silvestri S. De, Laporta P. and Svelto O., Opt. Lett. 9 (1984) 333.

4. Laporta P. and Magni V., Appl. Opt. 24 (1985) 2014

5. Fork R.L., Greene B.I. and Shank C.V., Appl. Phys. Lett. 38 (1981) 671.

6. Valdamis J.A., Fork R.L. and Gordon J.P., Opt. Lett. 10 (1985) 131.

7. Fork R.L., Shank C.V., Hirlimann C., Yen R. and Tomlinson W.J., Opt. Lett. 8 (1983) 1.

8. Know W.H., Fork R.L., Downer M.C., Stolen R.H., Shank C.V. and Valdamis, Appl. Phys. Lett. 46 (1985) 1120.

9. Ippen F.P. and Shank C.V. in: Ultrashort Light Pulses; ed. by S.L. Shapiro (Springer Verlag) 1977, p. 83.

10. Ultrafast Phenomena $I V$, ed. by D.H. Auston and K.B. Eisenthal (Springer Verlag) 1984.

11. Downer M.C., Fork R.L. and Shank C.V., J. Opt. Soc. Am. B 2 (1985) 595.

12. Weiner A.M., Silvestri S. De and Ippen E.P., J. Opt. Soc. Am. B 2 (1985) 654.

13. Silvestri S. De, Fujimoto J.G., Ippen E.P., Gamble E.B., Williams L.R. and Nelson K., Chem. Phys. Lett. 116 (1985) 146.

14. Fujimoto J.G., Silvestri S. De, Ippen E.P., Puliafito C.A., Margolis R. and Oseroff A., Opt. Lett. 11 (1986) 150.

15. Valdamis J.A. and Mourou G., IEEE J. Quantum Electron. OE-22 (1986) 69.

Fig. 5 - Scattered energy from optic phonons as a function of probe delay in $\alpha$-perylene crystal at low temperature. The beating is due to excitation of 80 and $104 \mathrm{~cm}^{-1}$ optic phonons.

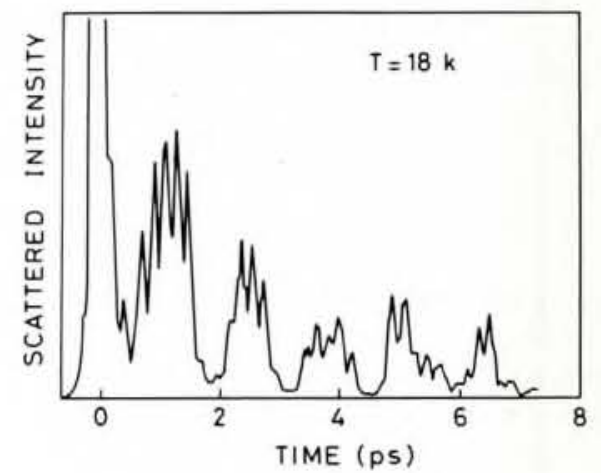

